\title{
Méthodologie pour prévoir le transfert des métaux lourds dans les chaînes trophiques marines incluant les mollusques filtreurs
}

\author{
H. Ettajani ${ }^{(1)}$, L. Pirastru ${ }^{(2)}$ \\ (1) Service d'Ecotoxicologie, URA 1356 CNRS, 1 rue G. Veil, 44035 Nantes Cédex (France) \\ (2) Laboratoire de Biologie Marine, URA 1356 CNRS, 2 rue de la Houssinière, 44072 Nantes \\ Cédex 01 (France) et IMC (International Marine Centre), Lungomare Eleonora d'Arborea, 22, \\ 09072 Torregrande Oristano (Italy).
}

Résumé. - La toxicité d'un métal à l'égard d'un organisme et sa biodisponibilité pour les niveaux trophiques supérieurs dépendent non seulement de son niveau global mais aussi de sa forme physico-chimique. Pour chaque niveau trophique, il convient donc d'examiner la répartition du métal entre formes solubles (métalloprotéines étudiées par des méthodes biochimiques) et insolubles (méthodes microanalytiques). La stabilité chimique des composés ainsi déterminés permet de prévoir leur activité biologique. Toutefois, avant que ce type de prévision puisse être généralisé il est indispensable de valider nos hypothèses en quantifiant effectivement le transfert des métaux entre les compartiments d'un modèle expérimental de chaîne trophique.

Notre démarche est illustrée à l'aide des résultats acquis sur le transfert du cuivre et de l'argent par voie directe (source eau de mer) ou particulaire (sédiment naturel, Diatomée $H$. ostrearia) vers l'Huître Crassostrea gigas. Les formes physico-chimiques de l'argent chez l'Huitre permettent de préjuger d'un transfert faible au consommateur. Pour le cuivre, le transfert par voie alimentaire n'est pas négligeable mais le risque encouru est vraisemblablement limité en raison du rôle biologique essentiel de ce métal et il convient de l'évaluer très précisément.

Mots-clés. - Chaîne alimentaire, Hữtre, Argent, Cuivre, Spéciation.

Abstract. - The bioavailability of metals in food chain and their toxicity to living organisms depend not only on their gross concentration but also on their physicochemical form. Thus at each level, it is necessary to examine its distribution among soluble forms (metalloproteins studied using biochemical techniques) or insoluble forms (using electron probe microanalysis). The chemical stability of the compounds thus identified permits to predict their biological activity. Before this approach can be widely generalized, however, it is essential to validate our hypotheses by quantifying real metal transfer between the compartments of a food chain model.

This approach is illustrated here by data acquired about copper and silver transfer to 
oysters (Crassostrea gigas) exposed through sea water or via particulate matter (natural sediment, diatoms). The physico-chemical forms of silver stored in oysters suggests a moderate transier to consumers. In the case of copper, transfer through feeding is not a negligible factor, through the associated risk is not doubt limited because of the key biological role played by this metal, and it therefore merits careful evaluation.

Key word. - Food chain, Oyster, Silver, Copper, Speciation.

\section{INTRODUCTION}

L'industrie et les ressources marines sont deux activités essentielles pour l'économie marocaine. Cependant, un conflit d'utilisation s'installe entre l'industrie qui met en jeu des métaux lourds, et l'aquaculture développée en zônes côtières sujettes aux rejets de polluants. Aussi est-il nécessaire de prévoir le devenir des métaux lourds au niveau du littoral.

La concentration globale d'un polluant métallique au niveau des organismes est souvent considérée comme indicateur de sa toxicité potentielle. Cependant un effet biologique ou écologique ne suit pas nécessairement l'accumulation des métaux par les organismes marins. En effet chez les organismes manifestant un pouvoir de bioconcentration élevé des polluants, tels que les mollusques filtreurs (Huitre et Moule) internationalement retenus comme indicateurs biologiques de pollution, il existerait des mécanismes qui permettent de séquestrer le métal sous des formes physico-chimiques peu ou pas toxiques, pouvant être disponibles ou non aux maillons suivants de la chaîne trophique.
Selon Campbell et al., (1988) un métal est considéré comme biologiquement disponible quand il peut être pris par un organisme et peut réagir avec sa machinerie métabolique. Plusieurs facteurs peuvent contrôler cette biodisponibilité : a) la spéciation chimique des métaux tant au niveau du vecteur de contamination qu'au niveau de l'organisme contaminé; b) les caractéristiques spécifiques de la matière en suspension qui représente le "point d'entrée" des éléments métalliques dans les chaines trophiques marines à mollusques filtreurs. Des études ont montré que des paramètres comme la taille et la nature (organique ou inorganique) des particules peuvent favoriser ou non leur utilisation par le maillon suivant et, par conséquent, le transfert, dans la chaîne trophique des polluants qui leurs sont associés (In: Campbell et al., 1988).

II est donc intéressant pour toute prévision d'effet biologique ou de transfert éventuel des éléments métalliques dans les chaines trophiques marines d'estimer la biodisponibilité de ces derniers pour chaque maillon trophique. Nous avons développé une méthodologie pour étudier le transfert de micropolluants dans une chaîne 
alimentaire comprenant : eau - particules en suspension (inertes ou vivantes) - bivalves - mammifères.

L'importance relative des deux vecteurs de contamination (eau, nourriture) pour les bivalves a été étudiée expérimentalement du fait que la concentration globale des métaux au niveau d'organismes contaminés in situ n'informe pas sur la contribution relative de ces deux vecteurs dans l'accumulation du polluant.

\section{LA PHASE PARTICULAIRE EN SUSPENSION}

La phase particulaire en suspension dans les milieux aquatiques (Algues, Bactéries, particules inertes organiques ou inorganiques) constitue la base des réseaux trophiques et d'autre part, présente souvent une grande capacité d'accumulation à l'égard de nombreux polluants dispersés dans l'environnement. Cette phase apparaît donc comme un vecteur potentiel de transfert de ces polluants dans les chaînes alimentaires. II convient d'élucider ce rôle, ce qui nécessite d'évaluer quantitativement le pouvoir contaminant des différents composants de la phase particulaire. Cette évaluation ne peut être abordée de façon convenable que si l'on dispose de particules dont les caractéristiques (taille et niveaux de contamination en particulier) sont parfaitement connues et dont les quantités sont suffisantes pour que la reproductibilité des doses de contaminants distribuées aux mol- lusques filtreurs soit assurée, dans des expériences conduites à moyen ou long terme.

Dans le cas de notre modèle expérimental de chaîne trophique, la phase particulaire est constituée de particules vivantes (Diatomée Haslea ostrearia) et de sédiment naturel riche en particules organiques susceptibles d'être digérées à l'égal des particules organiques vivantes.

\section{a) La phase particulaire inerte}

D'après les travaux de Sornin et al. (1988) des données précises sur l'utilisation d'argiles pures par l'Huître Crassostrea gigas Thunberg, montrent que les particules de taille inférieure à $2,5 \mu \mathrm{m}$ (illite) ne sont pas retenues efficacement par le Bivalve, celles de taille supérieure à $5 \mu \mathrm{m}$ (montmorillonite) sont rejetées sous forme de pseudofèces. La kaolinite de taille intermédiaire (3-4 $\mu \mathrm{m})$ apparaît comme l'argile la mieux adaptée comme vecteur de contaminants à l'égard des Bivalves.

Les particules les plus fines du sédiment naturel choisi ont été sélectionnées par élutriation. Cependant après remise dans l'eau de mer, $80 \%$ des particules formées ont une taille comprise entre 4,37 et $23,69 \mu \mathrm{m}$ avec une moyenne de $11,38 \mu \mathrm{m}$ (granulométrie Laser). En se basant sur les données mentionnées ci-dessus, il paraissait que des particules de cette taille ne seraient pas filtrées par l'Huître $C$. gigas. Toutefois il est connu que les huitres sélectionnent les particules 
disponibles en fonction de leur nature organique ou minérale (Deslous-Paoli, comm. pers.). Une expérience annexe a montré que ces particules riches en matière organique ont été effectivement ingérées par les huîtres.

L'affinité des particules sédimentaires à l'égard des micropolluants, détermine la quantité globale de micropolluants susceptible d'être ingérée par les mollusques filtreurs, ainsi que leur biodisponibilité. Selon Kersten et Förstner (1989), les fractions échangeables du métal sont plus importantes dans les particules en suspension que dans les sédiments déposés, ce qui induirait un relarguage relativement aisé des métaux dans le milieu, et par conséquent la diminution de la quantité susceptible d'être réellement transférée aux mollusques filtreurs par cette voie particulaire. Nous avons retrouvé un phénomène similaire en milieu expérimental lorsque les huîtres ont été exposées aux particules inertes contaminées par le cuivre (toutefois le métal libéré dans l'eau reste disponible pour une éventuelle bioaccumulation par voie directe).

Après ingestion du sédiment contaminé par le Mollusque filtreur, les phénomènes de la digestion joueront un rôle considérable dans l'extraction des éléments traces et ce d'autant plus que la présence des particules dans le tube digestif sera plus longue. L'extraction digestive est d'autant plus efficace que la taille des particules est plus faible (Campbell, 1988).

Des auteurs ont montré que la biodisponibilité de certaines formes du métal lié aux particules peut être in- versement proportionnelle à la force de la liaison métal-particule (Luoma \& Jenne, 1977). De ce fait certaines fractions du métal peuvent être plus "disponibles" que d'autres. Tessier et al. (1979) ont mis au point une méthode dite "d'extraction fractionnée" qui consiste en des attaques de plus en plus "agressives" du sédiment au moyen de réactifs différents, ce qui permet d'examiner la distribution des éléments traces au niveau des particules sédimentaires. Ces extractions ont un intérêt direct limité dans la mesure où elles ne peuvent pas mimer les systèmes biologiques mis en jeu dans les processus de résorption et d'extraction des éléments traces adsorbés aux niveau des particules. Elles restent cependant un outil efficace pour estimer la biodisponibilité des polluants: chez Nereis diversicolor par exemple, il a été montré que les niveaux d'argent, de cadmium, de cobalt et de zinc étaient mieux corrélés avec les concentrations dans certains extraits (acétate d'ammonium $1 \mathrm{~N}$ pour $\mathrm{Zn}, \mathrm{HCl} 1 \mathrm{~N}$ pour les autres métaux) qu'avec les concentrations globales dans le sédiment. Par contre, la disponibilité du plomb est aussi bien corrélée aux concentrations totales dans le sédiment qu'aux concentrations extractibles (Luoma, 1983). Les extractions fractionnées peuvent également être d'une utilité indirecte considérable pour 1) établir la capacité de liaison du métal à la particule ; 2) connaissant la distribution du métal au niveau du sédiment, prévoir sa réponse la plus probable aux changements des conditions physico-chimiques tels l'acidification ou les dragages. 


\section{b) La phase particulaire vivante}

En ce qui concerne les microorganismes, la prise d'un métal par ces derniers peut modifier effectivement sa biodisponibilité à l'égard des organismes du maillon suivant. Flatau et Gauthier (1983) ont montré que le cadmium fourni aux moules (Mytilus edulis) par l'intermédiaire de souches bactériennes préalablement cultivées sur milieu cadmié, était plus accumulé par les organismes que le cadmium ionique. De même, chez les huîtres l'accumulation du mercure augmente en présence de bactéries ayant accumulé ce métal : celles-ci en effet, effectuent une méthylation du mercure inorganique sous forme de méthylmercure le rendant plus assimilable par les organismes (Colwell et al., 1975; 1976; 1977; Berk et al., 1978).

Bryan (1984) a souligné l'influence importante de la nature de la matrice nutritive dans l'accumulation des métaux chez les Mollusques. Or, il a été montré que l'exposition à des polluants métalliques peut modifier la valeur nutritive de certains organismes phytoplanctoniques. Ainsi, la contamination de la microalgue Tetraselmis suecica par l'argent et le cuivre peut induire une perturbation au niveau du système enzymatique de stockage des polysaccharides, ce qui entraînerait une réduction au niveau du sucre de ce nutriment particulaire (BallanDufrançais et al., in press).

Dans le cas de notre modèle expérimental, nous avons étudié "la qualité " d'H. ostrearia après contami- nation par différentes concentrations d'argent et de cuivre. L'influence de ces concentrations aussi bien sur le plan quantitatif (coefficient de croissance des algues), que sur le plan qualitatif (teneurs en azote et en carbone particulaire, teneurs en pigments totaux et en marennine) a été examinée (Fig. 1 et 2).

Un test d'interaction entre l'argent et le cuivre a été effectué. Ces interactions entre polluants peuvent conduire éventuellement à une exaltation (synergie) ou à une limitation (antagonisme) de leurs effets toxiques individuels, phénomène que nous avons retrouvé chez $H$. ostrearia (Fig. 2).

Le choix des niveaux d'exposition d'H. ostrearia à l'argent et au cuivre était délicat dans la mesure où il a fallu respecter plusieurs impératifs. Ces niveaux devaient être: 1) compatibles avec les niveaux susceptibles d'être rencontrés dans les environnements pollués; 2) compatibles avec le développement d'une biomasse algale suffisante pour constituer des stocks de nourriture phyto- planctonique conséquents; 3 ) induire un niveau d'imprégnation des algues suffisant pour qu'un éventuel transfert au consommateur soit mesurable. Pour Skeletonema costatum, des concentrations de $20 \mu \mathrm{g} \mathrm{Ag} / \mathrm{l}$ et $50 \mu \mathrm{g}$ $\mathrm{Cu} / \mathrm{l}$ répondaient à ces exigences (Martoja et al., 1988 ; Amiard et al., 1989). II en est de même pour T. suecica. Cependant $H$. ostrearia a manifesté une sensibilité plus grande aux deux métaux ce qui nous a conduit à choisir des niveaux d'exposition rela- 
Concentration on $\mathrm{Cu}(\mu \mathrm{g} / \mathrm{g})$
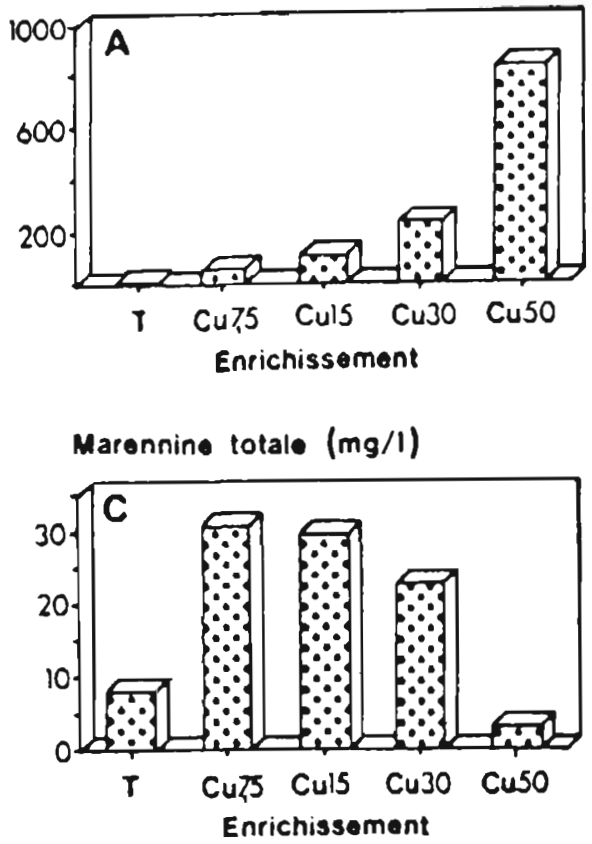

N particulaire (mg/l)

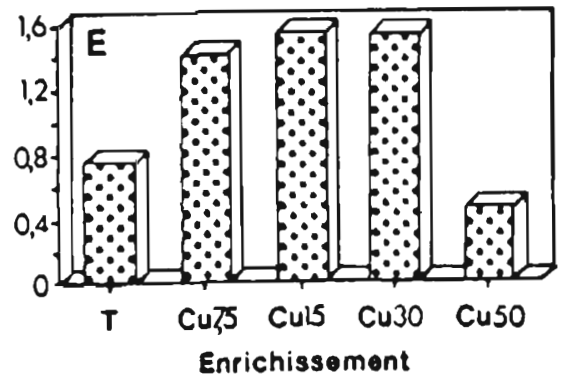

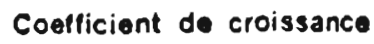

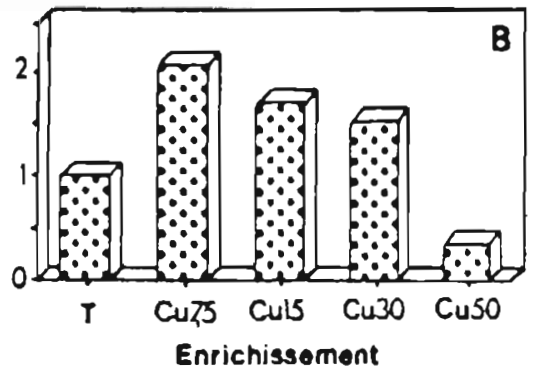

Pigments totaux $(\mu / / 1)$

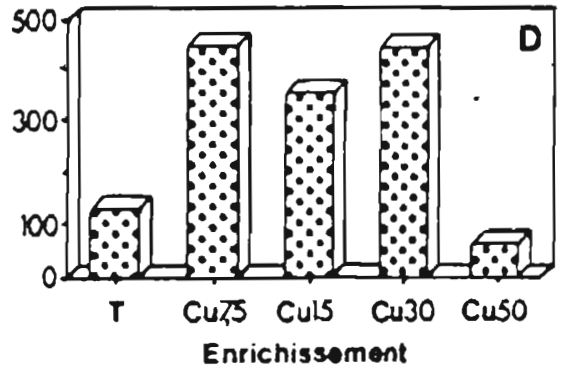

C particulair• (mg/l)

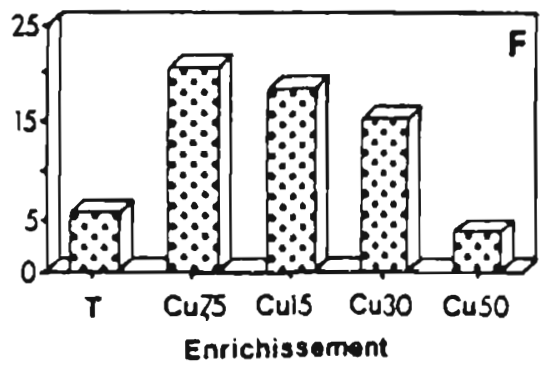

Fig. 1. - Bioaccumulation et effets biologiques du cuivre à l'égard de la Diatomée Haslea ostrearia cultivée sur un milieu ayant reçu des enrichissements croissants de ce métal ( $T$ : témoin; $\mathrm{Cu} 7,5$ : milieu enrichi en cuivre à $7,5 \mu \mathrm{g} / \mathrm{l})$.

A - Concentration du cuivre dans les algues. B - Infuence sur la croissance (le coefficient de croissance est le rapport entre le nombre de cellules dans un milieu donné et le nombre de cellules dans le milieu témoin): $C$ à $F$ - Infuence sur la composition de la biomasse algale.

Fig. 1. - Bioaccumulation and biological effects of copper to the diatom Haslea ostrearia grown in Cu-enriched media (T: Control; $\mathrm{Cu} 7,5 \mathrm{:} \mathrm{Cu}$ overload in seawater $=7,5 \mu \mathrm{g} / \mathrm{l}$ ).

$A$ - Cu concentration in algae; B - Influence on growth (the growth coefficient is the ratio between the cell number in a given medium and the cell number in control); $C$ to $F$ - Influence on constituants of the algal biomass. 
Concentration on $\mathrm{Ag}(\mu \mathrm{g} / \mathrm{g})$
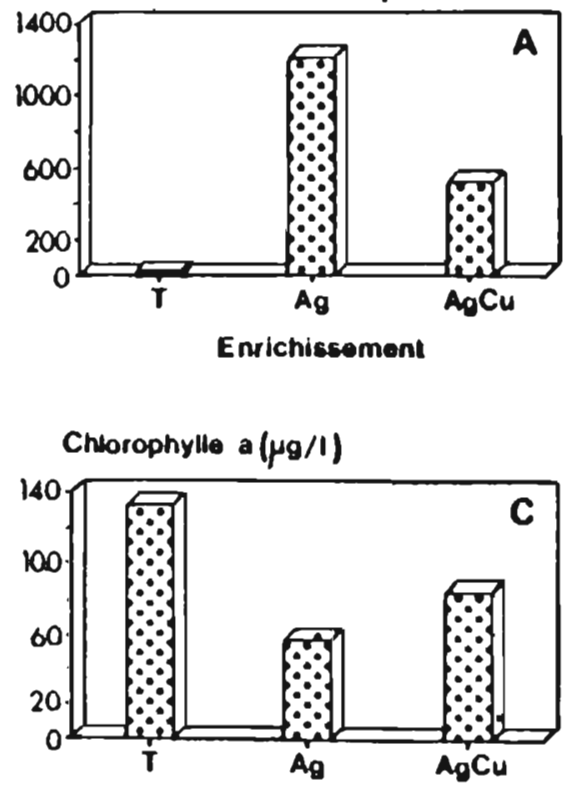

Envichissement
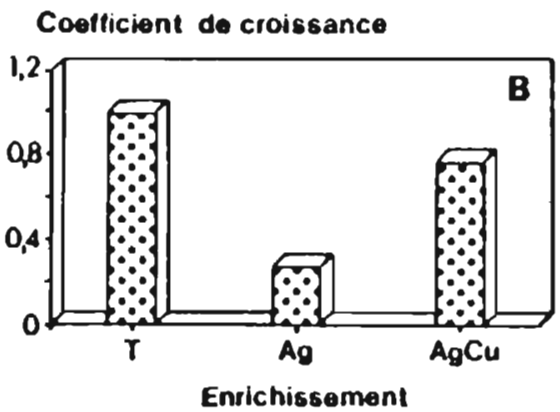

Marennine tot $(\mathrm{mg} / \mathrm{l})$

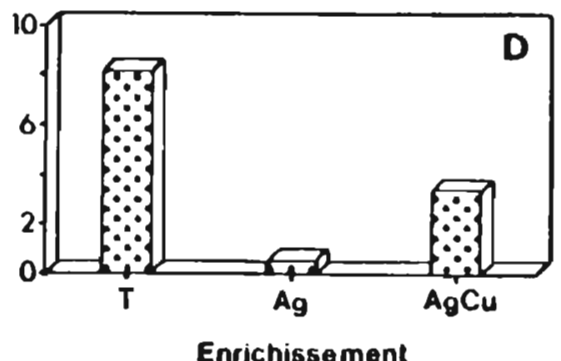

Fig. 2 - Bioaccumulation et effets biologiques de l'argent à l'égard de la Diatomée Haslea ostrearia exposée à ce métal seul $(20 \mu \mathrm{g} / \mathrm{l})$ ou en association avec le cuivre $(20 \mu \mathrm{g} / \mathrm{l}+50 \mu \mathrm{g} \mathrm{Cu} / \mathrm{l})$ ( $\mathrm{T}$ : témoin: $\mathrm{Ag}$ : milieu enrichi en argent; $\mathrm{AgCu}$ : milieu enrichi en argent et en cuivre).

A - Concentration de l'argent dans les algues. B - Influence sur la croissance (le coefficient de croissance est le rapport entre le nombre de cellules dans un milieu donné et le nombre de cellules dans le milieu témoin); C, D - Influence sur la composition de la biomasse algale.

Fig. 2. - Bioaccumulation and biological effects of silver to the diatom Haslea ostrearia exposed to this metal alone $(20 \mu \mathrm{g} / \mathrm{l})$ or mixed with copper $(20 \mu \mathrm{g} / \mathrm{l} \mathrm{Ag/l}+50 \mu \mathrm{g} \mathrm{Cu} / \mathrm{l})(\mathrm{T}$ : control; Ag: Ag-enriched medium; $\mathrm{Ag}$ - $\mathrm{Cu}$ : medium enriched with both $\mathrm{Ag}$ and $\mathrm{Cu}$ ).

A - Ag concentration in algae; B - Influence on growth (the growth coefficient is the ratio between the cell number in a given medium and the cell number in control); C, D - Influence on constituants of algal biomass.

tivement plus faibles $(10 \mu \mathrm{g} \mathrm{Ag/l} \mathrm{;} \mathrm{lié} \mathrm{à} \mathrm{des} \mathrm{composées} \mathrm{moléculaires} \mathrm{tels}$ $30 \mu \mathrm{g} \mathrm{Cu} / \mathrm{l})$.

La disponibilité du métal pour le consommateur est différente selon que le métal est seulement adsorbé au niveau de la surface algale ou pénétrant dans la cellule - se trouve que les phytochélatines. (Grill et al., 1987). Un rinçage des algues préalablement contaminées par des agents complexants spécifiques permet de décrocher le métal adsorbé à la surface algale (par exemple le 8- hydro- 
xyquinoline-5-sulphonate $1 \mathrm{mM}$ utilisé par Price et Morel (1990) pour la Diatomée Thalassiosira weissflogii contaminée par Co, Cd ou $\mathrm{Zn}$ ).

$\mathrm{Au}$ cours d'un colloque international sur l'écotoxicologie du sédiment (SEFA, 1991), plusieurs auteurs ont souligné l'importance de la phase dissoute par rapport à la phase particulaire dans la bioaccumulation de plusieurs polluants par les organismes aquatiques. Une étude expérimentale sur l'importance des deux voies dans l'accumulation de l'argent et du cuivre chez l'Huître $C$. gigas a montré qu'à court terme (2 à 3 semaines) cette espèce accumulerait plus d'Ag et de $\mathrm{Cu}$ à partir de la phase dissoute que de la phase particulaire. Toutefois le pourcentage de métaux retenu à partir des particules en suspension (algues ou sédiment) distribuées aux huîtres était toujours plus élevé que la fraction incorporée à partir de la phase dissoute (Ettajani et Amiard, in : SEFA, 1991). Ceci montre qu'à long terme la voie alimentaire jouerait un rôle non négligeable dans le transfert des éléments traces.

\section{BIOACCUMULATION CHEZ LES BIVALVES}

La prise en considération du vecteur de contamination est très importante quant à l'estimation de la biodisponibilité des polluants au niveau du mollusque filtreur. Des études expérimentales (Berthet, 1990) ont montré que selon le vecteur de conta- mination (eau ou nourriture), la spéciation chimique de l'argent au niveau des tissus de l'Huitre est différente.

Chez les Invertébrés et en particulier chez les Mollusques bivalves, la bioaccumulation des métaux se fait selon deux voies principales aboutissant à la liaison des éléments soit sous forme de métalloprotéines, soit sous forme de concrétions minérales. Parmi les métalloprotéines, les protéines apparentées à la métallothionéine (protéine de faible poids moléculaire ; 6 à $10 \mathrm{KDa}$, riche en cystéine, thermostable) semble jouer un rôle de séquestration efficace pour un certain nombre de métaux ( $\mathrm{Ag}, \mathrm{Au}$, $\mathrm{Cd}, \mathrm{Cu}, \mathrm{Hg}, \mathrm{Zn}$ ) et dans une large gamme de concentrations tissulaires (Nolan et Duke, 1983; Jenkins et Brown, 1985; Viarengo et al., 1985). Des concrétions inorganiques sont également susceptibles de lier différents métaux au niveau de cellules variées présentes dans différents tissus ou organes. Chez les Bivalves, ces concrétions inorganiques sont fréquentes au niveau du rein par exemple chez des Pectinidés (Carmichael et al., 1979; George et al., 1980; George, 1982), chez Pinna nobilis (Ghiretti et al., 1972), Yoldia thraciaeformis (Brand et al., 1984), Mytilus edulis (George et Pirie, 1980), Donax trunculus (Mauri et Orlando, 1982), ...

Pour le métal que nous avons particulièrement étudié, l'argent chez l'Huitre, nous retrouvons ces deux types de bioaccumulation sous forme organique et inorganique. L'Huitre $C$. gigas contaminée par l'argent dissous dans l'eau et/ou incorporé à la nour- 
riture, accumulait le métal essentiellement sous la forme inorganique. Cette dernière a été déterminée comme étant du sulfure d'argent qui est une forme insoluble très stable (Martoja et al., 1988). Quant aux formes organiques solubles de l'argent, elles sont liées à deux composés protéiques ( $\alpha$ et $\beta$ ) dont la masse moléculaire est respectivement de $300 \mathrm{KDa}$ et de 20 à $28 \mathrm{KDa}$.

L'importance relative de ces deux composés organiques liant l'argent est différente selon que l'Huître est contaminée via l'eau et/ou via la nourriture. En effet, l'importance du composé ( $\beta$ ) augmente lorsque le vecteur eau intervient dans la contamination, alors que lors d'une contamination par voie alimentaire, le composé $(\alpha)$ est plus important. Dans le cas d'une contamination mixte, le pourcentage d'argent soluble est plus élevé dans le composé de faible masse moléculaire $(\beta)$ que dans celui de haute masse moléculaire $(\alpha)$.

Une fraction importante de l'argent étant immobilisée sous une forme physico-chimique stable (sulfure d'argent), tel qu'elle ne soit pas dégradée au cours du transit digestif, le transfert éventuel se trouverait limité. Par contre, la présence dans les mollusques d'autres composés solubles tels que les métalloprotéines, demande une évaluation plus fine du risque de transfert, pouvant aller jusqu'à la mise en place d'expériences d'alimentation de petits mammifères à partir de mollusques. Ceci permettrait de voir la répartition du métal au niveau du mammifère et d'estimer les entrées et les sorties du polluant (urine, fecès, ... etc.).

Plus généralement comme l'ont souligné Martoja et Martoja (1984), un métal associé à une métalloprotéine est biodisponible puisque la protéine peut être détruite par les enzymes digestives alors qu'il ne l'est pas s'il est engagé dans une combinaison qui reste stable malgré la forte acidité du suc gastrique (par exemple le cuivre présent sous forme de sulfure chez les gastéropodes, Bouquegneau et Martoja, 1982; le mercure présent sous forme de tienmanite chez les mammifères marins, Martoja et Berry, 1980).

\section{CONCLUSION}

La complexité des phénomènes relatifs à la contamination des systèmes naturels est liée à la multitude des actions et des interactions entre les différents facteurs abiotiques, biotiques et de contamination. Ces derniers sont relativement plus contrôlés dans un modèle expérimental. Cependant, un modèle trop simplifié, s'éloignant des conditions prévalant dans le milieu naturel ne reflète pas fidèlement les phénomènes naturels. Dans le cas de notre modèle, les niveaux métalliques d'exposition ont été choisis afin de ne pas provoquer de perturbations du développement des cultures algales ou de la physiologie des mollusques, mais également de maintenir dans le milieu externe des concentrations "réalistes" par rapport à celles 
qui peuvent être rencontrées dans les milieux les plus pollués.

Les modèles expérimentaux permettent de prendre en considération plusieurs facteurs et, entre autres de discerner la part des différentes voies d'exposition dans l'incorporation des polluants. Les études in situ n'autorisent pas cette distinction mais permettent par contre de s'affranchir de toutes les difficultés d'interprétation associées à la simplification des modèles expérimentaux. Dans l'un et l'autre type d'approche il est possible d'examiner la spéciation chimique des polluants au niveau des différents maillons de la chaîne trophique.

De l'analyse des formes physicochimiques sous lesquelles se présente le polluant, il est possible d'évaluer avec une probabilité raisonnable sa biodisponibilité et par conséquent de prévoir son devenir dans les chaînes trophiques dont le dernier maillon pourrait être l'Homme. Toutefois la fiabilité d'une telle prévision repose sur la représentativité des organismes choisis au sein du réseau trophique et nécessite une connaissance approfondie de la structure et du fonctionnement des écosystèmes côtiers.

\section{RÉFÉRENCES BIBLIOGRAPHIES}

Amiard J-C., Amiart-Triquet C., Ballan-Dufrançais C., Berthet B., Jeantet A.Y., Martoja R. \& Truchet M., 1989. Study of the bioaccumulation at the molecular, cellular and organism levels of lead and copper transferred to the oyster
Grassostrea gigas Thunberg directly from water or via food. Proc. 21st EMBS, Gdansk, 14-19 sept. 1986, Polsh. Acad. Sci., Inst. Oceanol., ed.: 521-529.

Berk S.G., Mills A.L., Hendricks D.L. \& Colwell R.R., 1978. Effects of ingesting mercury-containing bacteria on mercury tolerance and growth rates of ciliates. Microbial Ecol., 4 : 319-330.

Berthet B., 1990. Influence de la voie de contamination sur les formes physicochimiques de l'argent chez Crassostrea gigas Thunberg. Océanis, 16 (5): 349-357.

Bouquegneau J.M. \& Martoja M., 1982. La teneur en cuivre et son degré de complexation chez quatre Gastéropodes marins. Données sur le cadmium et le zinc. Oceanol. Acta, 5 (2): 219-228.

Brand D.G., Farrell M.A. \& Reid B.G., 1984. Preliminary histological assessment of heavy metal accumulation in the bivalve Yoldia thraciaeformis collected from Alice Arm, Hastings Arm and Satellite Channel, B.C. Can. Ms. Rep. Fish. Aquat. Sci. 1770: vii +32 p.

Bryan G.W., 1984. Pollution due to heavy metals and their compounds. In: Marine Ecology, Kinne O., ed., John, Wiley \& Sons LTD, New-York, 5 (3): 1289-1431.

Campbell P.G.C., Lewis A.G., Chapman P.M., Crowder A.A., Fletcher W.K., Imber B., Luoma S.N., Stokes P.M. \& Winfrey M., 1988. Biologically available metals in sediments. Publications, NRCC/CNRC Ottawa (Canada) Pub. $N^{\circ}$. NRCC 27694298 p.

Carmichael N.G., Squibb K.S. \& Fowler B.A., 1979. Metals in the molluscan kidney: a comparison of two closely related bivalve species (Argopecten) using $X$-ray microanalysis and atomic absorption spectroscop. J. Fish. Res. Boad Can., 36: 1149-1155.

Colwell R.R., Sayler G.S. \& Nelson J.R., 1985. Role of bacteria in bioaccumu- 
lation of mercury in the oyster App. Microbiol., 30: 91-96.

Colwell R.R., Sayler G.S., Nelson J.D. \& Justice A., 1976. Microbial mobilization of mercury in the aquatic environment. In Environmental Biogeochemistry. Vol. 2. J. O. Nriagu, ed., Ann. Arbor. Science Pub., Ann Arbor, MI.: 473487.

Colwell R.R., Berk S.G., Sayler J.S., Nelson J.D. \& Esser J.M., 1977. Mobilisation of mercury by aquatic microorganisms. Proc. Int. Conf. on Heavy metals. Vol. 2: 832-843.

Flatau G.N. \& Gauthier M.J., 1983. Accumulation du cadmium par Mytilus edulis en présence de souches bactériennes sensibles ou résistantes à ce métal. Can. J. Microbiol., 29: 210217.

Ghiretti F., Salvato B., Carlucci S. \& DePieri R., 1972. Manganese in Pinna nobilis. Experiencia, 28: 232-233.

Grill E., Winnacker E.L. \& Meinhard H.Z., 1987. Phytochelatins, a class of heavy-metal-binding peptides from plants, are functionally analogous to metallothioneins. Proc. Natl. Acad. Sci. USA 84: 439-443.

Jenkins K.D. \& Brown D.A. 1985. Determining the biological significance of the contaminant bioaccumulation. I $n$ : Concepts in Marine Pollution Mesurements, Harris $\mathrm{H}$. White, ed., Maryland Sea Grant College: 355-375.

Kersten M. \& Förstner U. 1989. Speciation of trace elements in sediment. In: Trace element speciation: analytical methods and problems, Batley G.E., ed. CRC Press, Boca Raton, FL, 8: 245-317.

Luoma S.N. \& Jenne E.A., 1977. The availability of sediment-bound cobalt, silver and zinc to a deposit-feeding clam. In Biological Implications of Metals in the environment. H. Drucker and R.E. Wildung, eds. ERDA Symp. Ser. No 42, Energy Research and Development Administration, Washington, D.C.: 213230.
Luoma S.N., 1983. Bioavailability of trace metals to aquatic organisms. A Review. Sci. Total Environ., 28: 1-22.

Martoja R. \& Berry J.P., 1980. Identification of tienmannite as a probable product of demethylation of mercury by selenium in Cetaceans. A complement to the scheme of the biological cycle of mercury. Vie et milieu, 30: 7-10.

Martoja R., Ballan-Dufrançais C., Jeantet A.Y., Gouzerh P., Amiard J.C., AmiardTriquet C., Berthet B. \& Baud J.P., 1988. Effets chimiques et cytologiques de la contamination expérimentale de l'Huitre Crassostrea gigas Thunberg par l'argent administré sous forme dissoute et par voie alimentaire. Can. J. Fish. Aquat. Sci., 45: 1827-1841.

Martoja M. \& Martoja R., 1984. La bioaccumulation de métaux, processus physiologique normal et conséquence de la pollution. Le courrier du CNRS, 54 : 32-37.

Mauri M. \& Orlando E., 1982. Experimental study on renal concretions in the wedge shell Donax Trunculus L. J. Exp. Mar. Biol. Ecol., 63: 47-57.

Nolan C.V. \& Duke E.J., 1983. Cadmium accumulation and toxicity in Mytilus edulis: Involvement of metallothioneins and heavy-molecular weight protein. Aquatic Toxicology, 4: 153-163.

Price N.M. \& Morel F.M.M., 1990. Cadmium and cobalt substitution for zinc in marine diatom. Nature, 344: 657660.

SEFA 1991. L'écotoxicologie des sédiments. Congrès International, La Rochelle, 5-7 juin 1991. Ed. Ministère de l'environnement.

Sornin J.M., Deslous-Paoli J.M. \& Hess O., 1988. Experimental study of the filtration of clays by the oyster Crassostrea gigas Thunberg: adjustement of particle size for best retention. Aquaculture, 69: 355-366.

Tessier A., Campbell P.G.C. \& Bisson M. 1979. Sequential extraction procedure 
for the speciation of particulate trace metals. Anal. Chem., 51: 844-851.

Viarengo A., Moore M.N., Pertica M., Mancinelli G., Zanicchi G. \& Pipe R.K.,
1985. Detoxification of copper in the cells of the digestive gland of mussel: the role of lysosomes and thioneins. Sci. Total Environ., 44: 135-145. 